\title{
Finite element analysis of the deformation behaviour of pure aluminium in repetitive corrugation-straightening and constrained groove pressing
}

\section{Farhad Rahimi}

Materials Processing Simulation Laboratory,

School of Metallurgy and Materials Engineering,

Iran University of Science and Technology (IUST),

Narmak, Tehran, Iran

Email: farhad.rhm@gmail.com

\section{Bagher Mohammad Sadeghi*}

School of Metallurgy and Materials Engineering,

Iran University of Science and Technology (IUST),

Narmak, Tehran, Iran

Fax: +98 (0) 21-77-240-480

Email: bmsadeghi@iust.ac.ir

*Corresponding author

\section{Masoud Ahmadi}

Department of Mechanical Engineering,

Bilkent University,

06800 Bilkent, Ankara, Turkey

Email: masoudahmadi777@gmail.com

\begin{abstract}
Repetitive corrugation-straightening (RCS) and constrained groove pressing (CGP) are two promising severe plastic deformation techniques for sheet metallic materials. There are differences in amount of imposed plastic strain, hence, it is important to compare the deformation mode in these two techniques. In the present paper, the effect of RCS and CGP on the deformation behaviour of pure aluminium is studied. The effective parameters are analysed using finite element analysis (FEA) for one pass of deformation. The computer simulations are carried using DEFORM-3D and to verify the FEA results, a comparison with previous literatures is made. It is observed that filling ratio of the die is higher for CGP. Additionally, the deformed work-piece endured more plastic strain in CGP and the effective strain is more homogenously distributed in this technique.
\end{abstract}

Keywords: severe plastic deformation; SPD; constrained groove pressing; CGP; repetitive corrugation and straightening; RCS; finite element analysis; FEA. 
Reference to this paper should be made as follows: Rahimi, F., Sadeghi, B.M. and Ahmadi, M. (2018) 'Finite element analysis of the deformation behaviour of pure aluminium in repetitive corrugation-straightening and constrained groove pressing', Int. J. Manufacturing Technology and Management, Vol. 32, No. 6, pp.598-609.

Biographical notes: Farhad Rahimi is a Research Assistant in Materials Processing Simulation Laboratory in School of Metallurgy and Materials Engineering at Iran University of Science and Technology. His research interests are mainly focused on severe plastic deformation, simulation of materials processing, sheet metal forming and areas related to mechanical properties of materials. He currently does research under the supervision of Dr. Mohammad Sadeghi in constrained groove pressing as a severe plastic deformation technique in production of ultrafine grained sheet materials both in experimental and numerical analysis.

Bagher Mohammad Sadeghi is an Assistant Professor in School of Metallurgy and Materials Engineering at Iran University of Science and Technology. He received his PhD from ENSAM University in France. His research interests lie in the area of metal forming, ranging from simulation to design and implementation. His recent research interests are mainly focused on severe plastic deformation, cupping and V-bending test, and full-field strain measurement using digital image correlation method. He teaches metal forming, continuum mechanics, theory of plasticity and mechanical properties of materials.

Masoud Ahmadi is a graduate student at Department of Mechanical Engineering in Bilkent University. His main research subject is studying multi-scale metal forming processes in both experimental and simulation investigations including severe plastic deformation and materials science issues including microstructure, phase transformation and etc. He is currently doing research on multi-scale machining of titanium alloys at Micro System Design and Manufacturing Center at Bilkent University. His undergraduate major was metallurgy and materials engineering at Iran University of Science and Technology. He studied spring-back phenomenon and phase transformation of 304L stainless steel during V-bending as his BSc thesis.

This paper is a revised and expanded version of a paper entitled 'Finite element simulation of the deformation behavior and homogeneity of AA 1070 aluminum in CGP and RCS' presented at 13th Asian Symposium on Precision Forging, Gyeongju, South Korea, 17-18 September 2015.

\section{Introduction}

Sheet materials are a major group of products which are widely used in important industries such as air or marine industries. Consequently, many researchers have focused their attentions on the modification of the mechanical and metallurgical properties of these materials (Khodabakhshi et al., 2010). Metal working results in enhancement of mechanical properties and hardness of metals after cycles of deformation (Hosford and Caddell, 1993). As a result, the workability of these processed products is reduced and limits the possibility of further processing. According to this limitation, many industrial processes cannot be performed on sheet metals to manufacture such desirable products 
(Valiev et al., 2000). Severe plastic deformation (SPD) is an effective technique through which relatively a large amount of cold working is applied on a metallic work-piece (Bisadi et al., 2010).

Based on the geometry of the processed work-piece, all SPD techniques are classified into three main categories; bulk, sheet and tube forming. Among all SPD sheet forming techniques, accumulative roll bonding (ARB) (Saito et al., 1998), constrained groove pressing (CGP) (Zhu et al., 2001) and repetitive corrugation and straightening (RCS) (Huang et al., 2001) have the possibility of industrial applications, consequently, these techniques are well under investigation.

The ARB method was introduced by Saito et al. in 1998. The main aim of this methodology is to apply plastic strain on sheet metallic materials during cold rolling. The process is performed in three steps, a sample is:

1 brushed and cleaned of any contaminations

2 cut into two equal pieces

3 faced on each other after preparation, afterwards, the rolling process will be done.

By repeating the above processes the mechanical properties of the material are increased and high level of grain refinement is achieved.

CGP is a new method of SPD for sheet materials which was introduced by Zhu et al. in 2001. In this process, the sample experiences four cycles of processing to complete one pass of deformation which contains two cycles of bending and the other two for straightening. The four cycles are (Zhu et al., 2001):

1 the work-piece is placed between the top and bottom constrained groove dies and pressed as shown in Figure 1(a)

2 the deformed work-piece is straightened by using two flat dies [Figure 1(b)]

3 the work-piece is rotated $180^{\circ}$ (clockwise or counter clockwise) around the pressing direction (see Figure 1) and placed between the grooved dies, this causes the non-deformed sections to be deformed by further processing [see Figure 1(c)]

4 the final cycle is to flatten the deformed work-piece by using two flat dies [Figure 1(d)].

The distance between top and bottom in both grooved dies is equal to the sheet thickness. So, it causes the bent areas of the sample to endure pure shear strain.

Figure 1 Schematic illustration of CGP technique

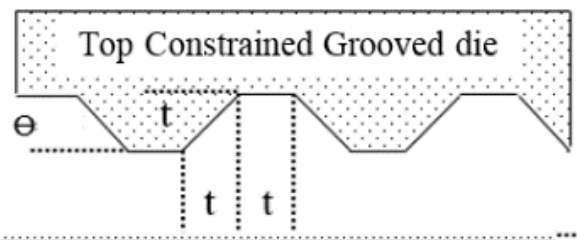

(b)

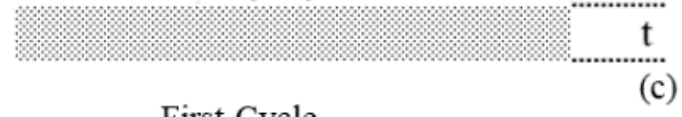

(a)

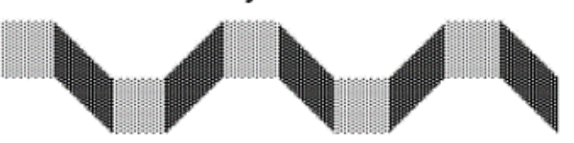

(d)

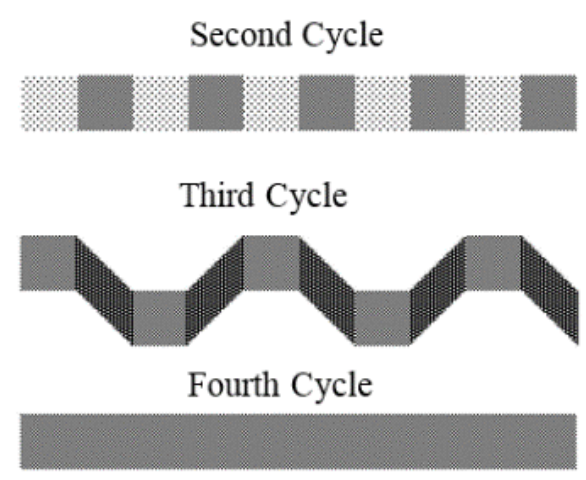

Source: Zhu et al. (2001) 
Figure 2 Schematic illustration of RCS technique, (a) bending (b) straightening
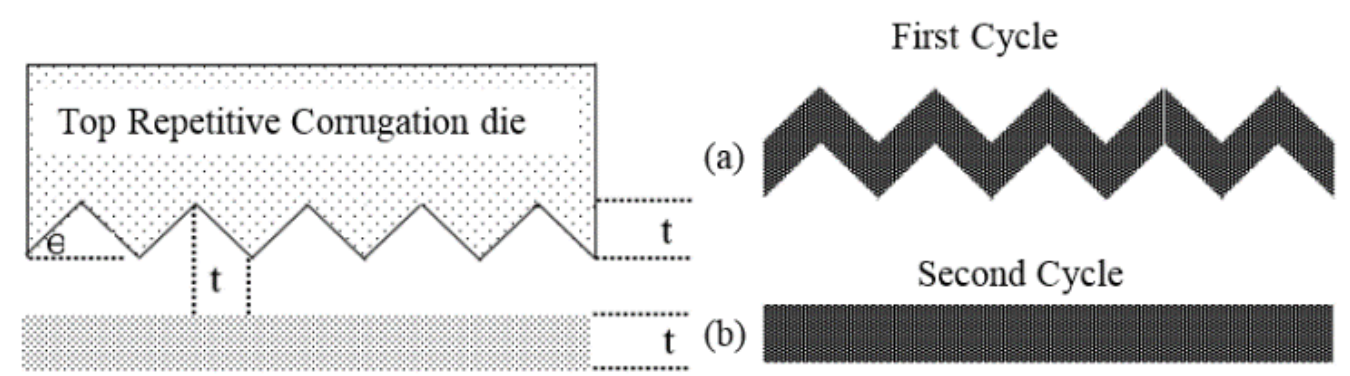

RCS was introduced for the first time by Huang et al. (2001) to produce ultra-fine grained sheets. In this method, sheets are subjected to bending and straightening cycles while dimensional alternations are negligible during deformation, consequently, the mechanical properties of the deformed sheet are increased. This process is schematically shown in Figure 2.

CGP and RCS have similarities in terms of processing cycles (bending and flattening) on the other hand, the values of imposed plastic strain are varied. Ideally, the processed work-piece should fully fill in the die channel in all SPD techniques. In that way, the sample attains its dimensional stability and as a result it provides the possibility of repetition of the process for more than one cycle to accumulate higher values of strain, consequently, high level of grain refinement will be achieved. The computer simulation and experimental results show that the deforming sample is not fully filled in the die channel and forms gaps (Talab and Ghalandari, 2011). Therefore, it is important to study the possibility of formation corner gaps in mentioned processes.

In the present study, the effect of CGP and RCS on the deformation behaviour of pure aluminium is investigated. For this purpose, the filling ratio of the die, the distribution of the effective strain and the required pressing load are evaluated after performing one complete pass of deformation by using finite element analysis (FEA). Inhomogeneity index of the effective strain distribution is used to estimate the homogeneity of deformation. The computer simulation is performed using DEFORM-3D ${ }^{\mathrm{TM}}$ software. A comparison between FEA results with other scientific results is used to validate the current numerical simulation.

\section{Finite element analysis}

The commercial finite element method (FEM) software, Deform $3 \mathrm{D}^{\mathrm{TM}}$, was used to perform the FEA of the deformation in RCS and CGP. The dies were modelled as rigid bodies. The work-piece was considered as samples with square cross section and dimensions of $10 \times 10 \times 1 \mathrm{~mm}^{3}$ with 30,000 elements. The die design parameters as presented in Figures 1 and 2 are listed in Table 1. Pure aluminium was selected as a sheet material and its characteristics that were implemented in the simulation are defined with the help of software manual (Scientific Forming Technologies Corporation, 2006) and illustrated in Figure 3.

The friction conditions between the surfaces of the sample and dies were considered as shear type with the coefficient of 0.1 . All computer simulations were performed at a punch speed of $0.1 \mathrm{~mm} \cdot \mathrm{s}^{-1}$ in isothermal conditions at room temperature. 
Table 1 Die design parameters used in the present study

\begin{tabular}{lcc}
\hline \multirow{2}{*}{ SPD technique } & \multicolumn{3}{c}{ Die design parameters } \\
\cline { 2 - 4 } & $\theta\left(^{\circ}\right)$ & $t(\mathrm{~mm})$ \\
\hline CGP & 45 & 1 \\
RCS & 45 & 1 \\
\hline
\end{tabular}

In order to investigate the effect of RCS and CGP on the homogeneity of strain and deformation, the inhomogeneity index $\left(C_{i}\right)$ is used and presented in equation (1) (Li et al., 2004):

$$
C_{i}=\frac{\varepsilon_{\max }-\varepsilon_{\min }}{\varepsilon_{a v g}}
$$

where $\varepsilon_{\max }, \varepsilon_{\min }$ and $\varepsilon_{a v g}$ are maximum, minimum and average effective strains, respectively. The effective strain distribution is considered homogenous at low value of $C_{i}$.

Figure 3 Flow stress of pure aluminium implemented in FEA (see online version for colours)

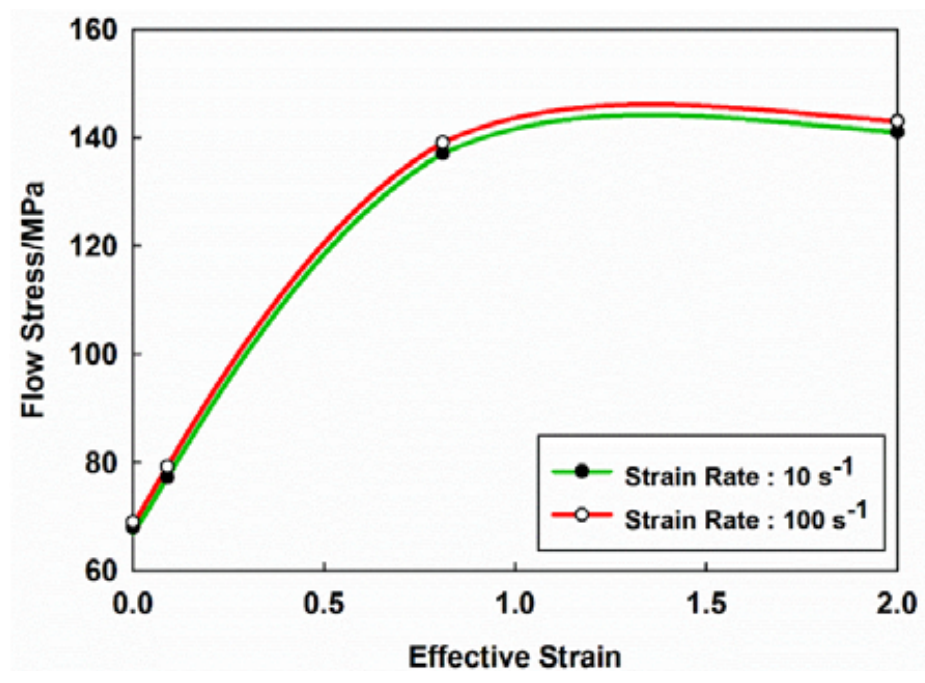

Source: Scientific Forming Technologies Corporation (2006)

\section{Results and discussion}

\subsection{FEA verification}

To verify the accuracy of the computer simulations, FEA results of CGP process are compared with the results of Ghazani and Vajd (2014). Figure 4 shows a comparison between inhomogeneity indices of the effective strain distributions achieved from FEA results and Ghazani after one pass of CGP processing. One may see that this figure shows a good agreement between the FEA and results of Ghazani. In that way, the conditions, i.e., coefficient of friction and upper die speed, that were implemented in the simulation for CGP is as similar as those are reported (Ghazani and Vajd, 2014). 
Figure 4 Comparison of the inhomogeneity index for FEA and Ghazani and Vajd (2014) results

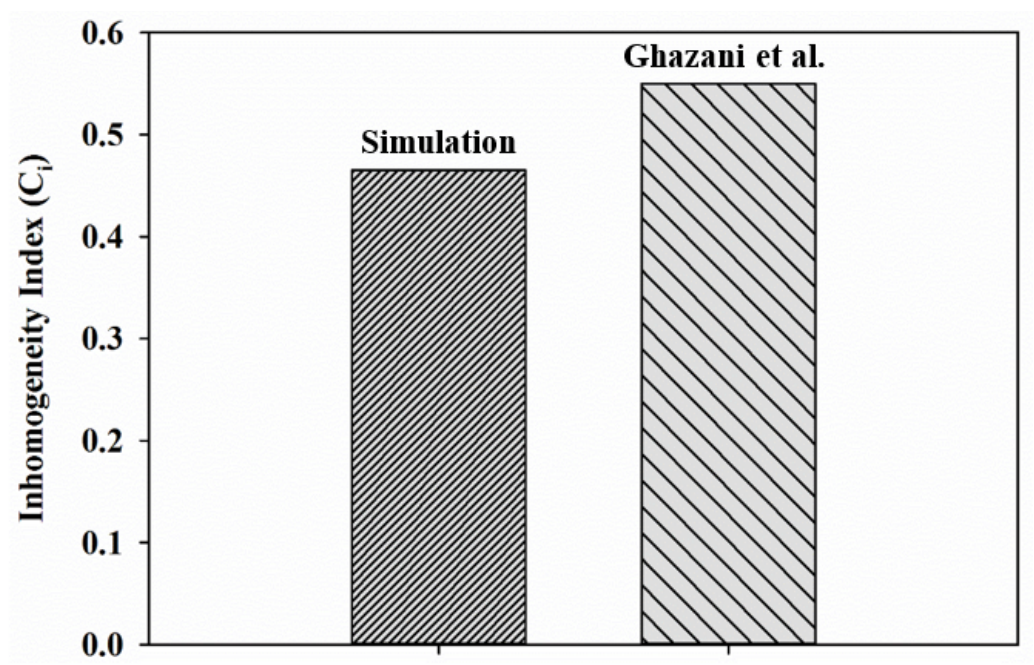

\subsection{Die filling ratio}

Ideally, the deformed samples by CGP and RCS processes should fully fill the die. In that way, this allows the sample to endure higher values of effective plastic strain. Thus, higher values of effective strain will be accumulated by repetition of these processes. On the other hand, the FEA results show that the sample does not completely fill in the die and gaps are formed as illustrated in Figure 5.

Figure 5 Illustration of the gaps formation in CGP and RCS, depicting gaps in red circles (see online version for colours)
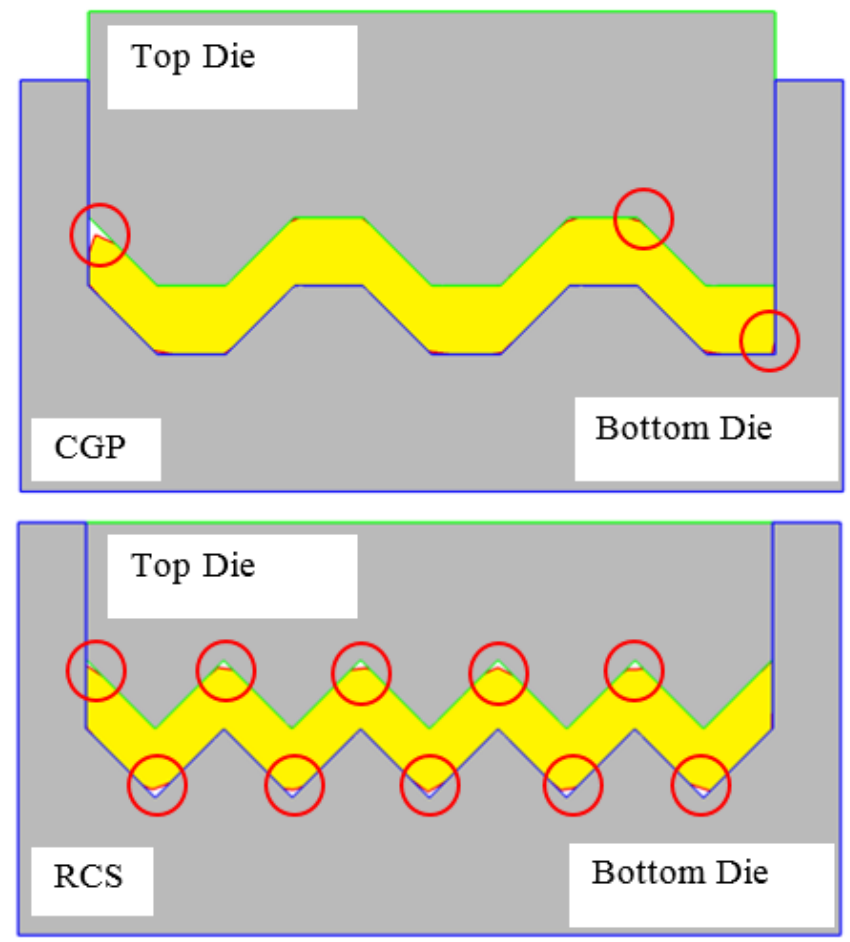

According to this figure, it can be seen that the possibility of formation of these gaps is significant in regions of sharp angles which is due to the presence of friction conditions between the surfaces of the work-piece and the die walls, thus, the work-piece prefers to 
consume less frictional energy during deformation and as a result, these gaps are formed in these sharp angles.

The filling ratio $\left(F_{R}\right)$ of the die is calculated and presented in equation (2) in order to evaluate the amount of gaps formation in the die channel after simulation of one pass of each techniques using ImageJ, an open source image processing software.

$$
F_{R}=\frac{S_{W}}{S_{C}} \times 100
$$

where $F_{R}$ is the filling ratio of the die, $S_{W}$ is the cross-sectional area of the work-piece and $S_{C}$ is the cross-sectional area of the die channel. The effect of the CGP and RCS on $F_{R}$ is shown in Figure 6. According to Figures 5 and 6, one may see that larger portions of the die are filled in CGP. Consequently, by repetition of CGP process, a higher value of effective plastic strain is imposed on the work-piece which leads to high level of grain refinement.

Figure 6 The die filling ratio for one cycle of CGP and RCS

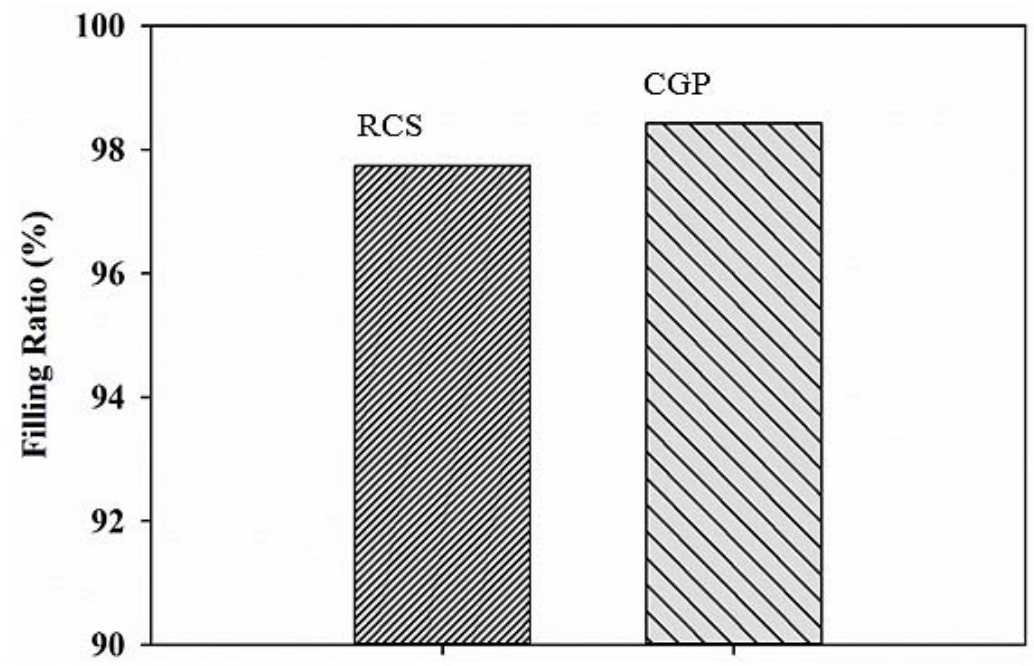

Figure 7 Average mean stress for the first cycle of deformation in CGP and RCS (see online version for colours)

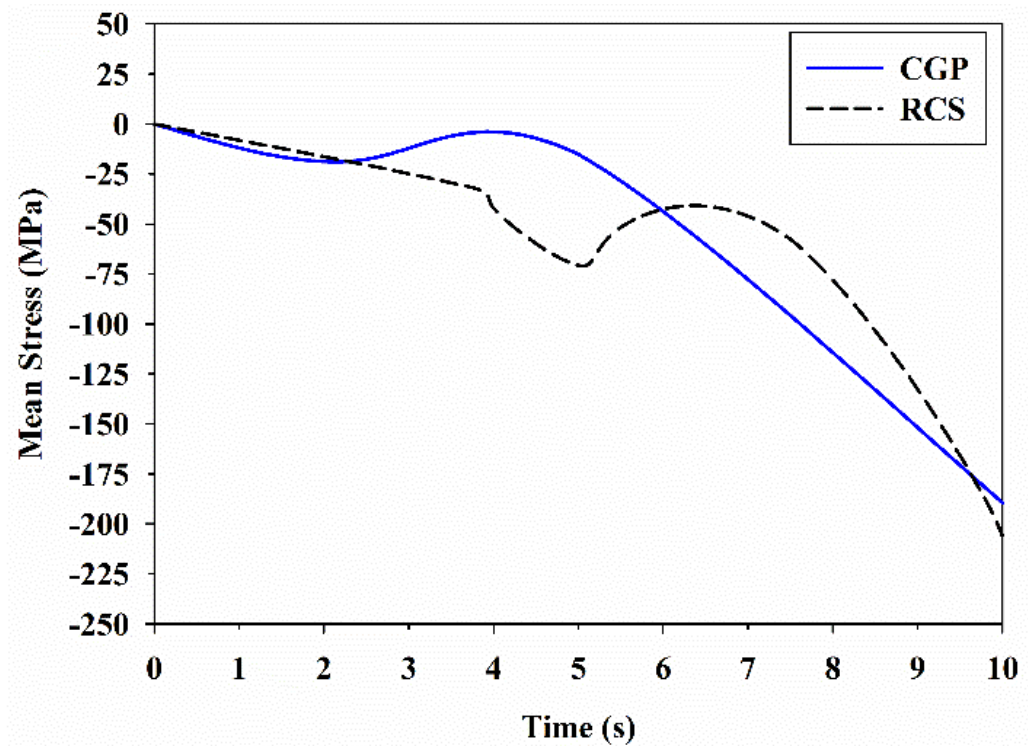




\subsection{Stress state}

Mean stress mode of deformed sample in the first cycle of deformation in CGP and RCS is shown in Figure 7. One may see that throughout these processes the sample almost experiences the same mean stress distribution. It is observed that during deformation for the first cycle, the sample endures higher values of compressive stress in RCS. Since, there are sharp dents with higher angle rather than CGP, the sample is sheared more severely and higher compressive stress is applied. Consequently, it is expected to impose higher plastic strain throughout this process and achieve finer grain structure as it is previously discussed by Huang et al. (2001).

\subsection{Effective strain distribution and deformation homogeneity}

The effective strain distribution was used in order to evaluate the amount of deformation applied to the work-piece. Figure 8 shows the imposed effective strain contour on the deformed work-piece in a plane alongside the pressing direction for the first cycle of CGP and RCS. In addition, Figure 9 shows the distribution fraction of the effective strain in these processes for one cycle of deformation. According to these figures, it is observed that in RCS the deformed sample endures higher strain at the sharp dents in contrast with CGP. This is due to the fact that in these regions, the material flow is severe during shear deformation which is related to the frictional contact areas that forces the work-piece to consume higher frictional energy during deformation. It is worth mentioning that the probability of crack formation at this regions (tip dents) in the deformed work-piece in RCS is higher. Thus, it can be concluded that the dents angle plays an important role on the distribution and for amount of effective strain.

Figure 8 Effective strain contour imposed on the work-piece (see online version for colours)

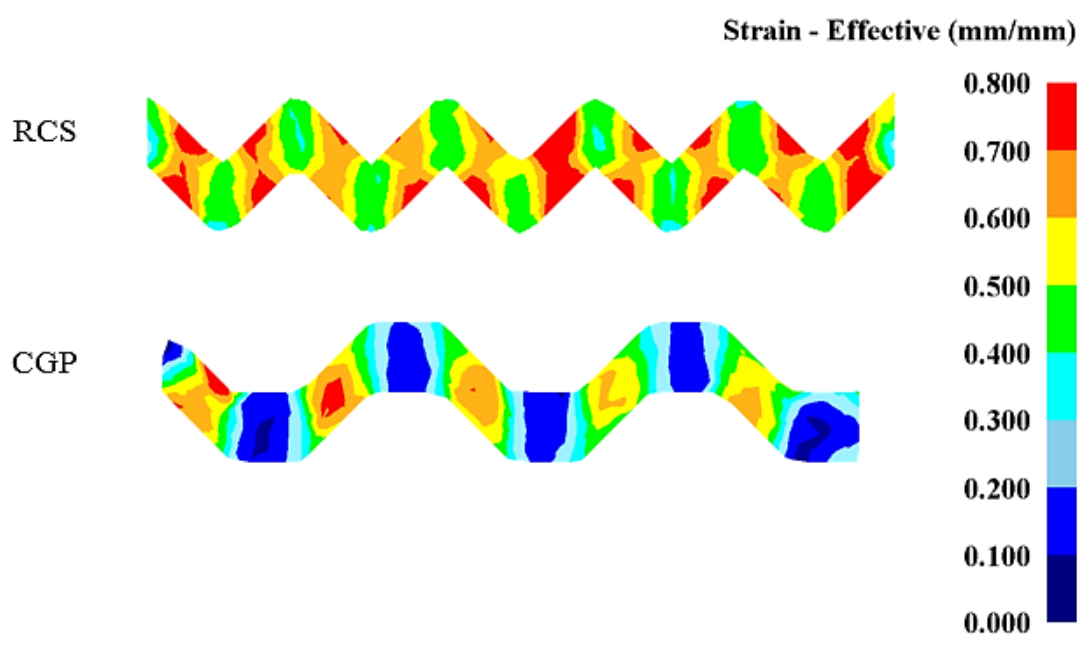

The effective strain maps in one dent for each technique are demonstrated in Figure 10. According to this figure, the deformed sample by RCS has endured severe strains due to existence of the sharp dents with the angle of $90^{\circ}$. In addition, in CGP a part of the deformed sample experienced shear strain and effective strain in these regions are lower than RCS which are approximately 0.7 and 0.8 , respectively. 
Figure 9 Distribution fraction of the effective strain in (a) RCS and (b) CGP (see online version for colours)

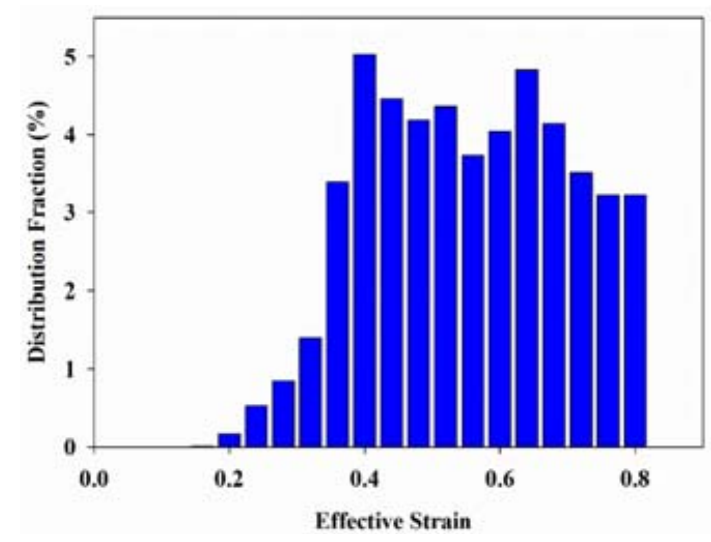

(a)

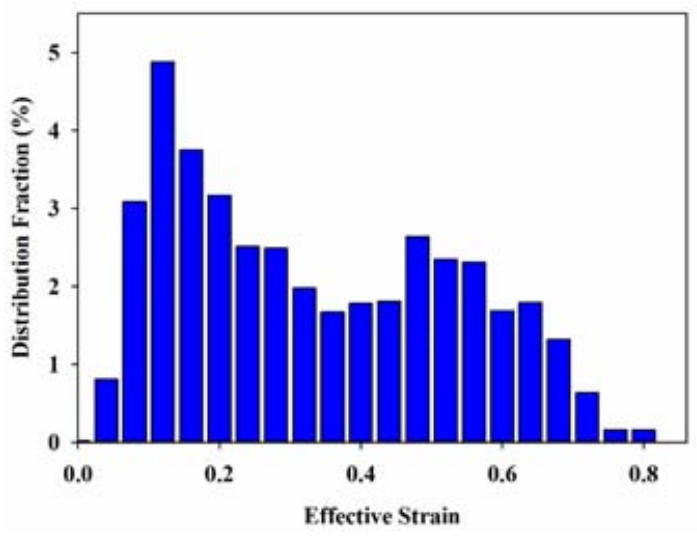

(b)

Figure 10 Variation of effective strain map for one cycle in CGP and RCS (see online version for colours)

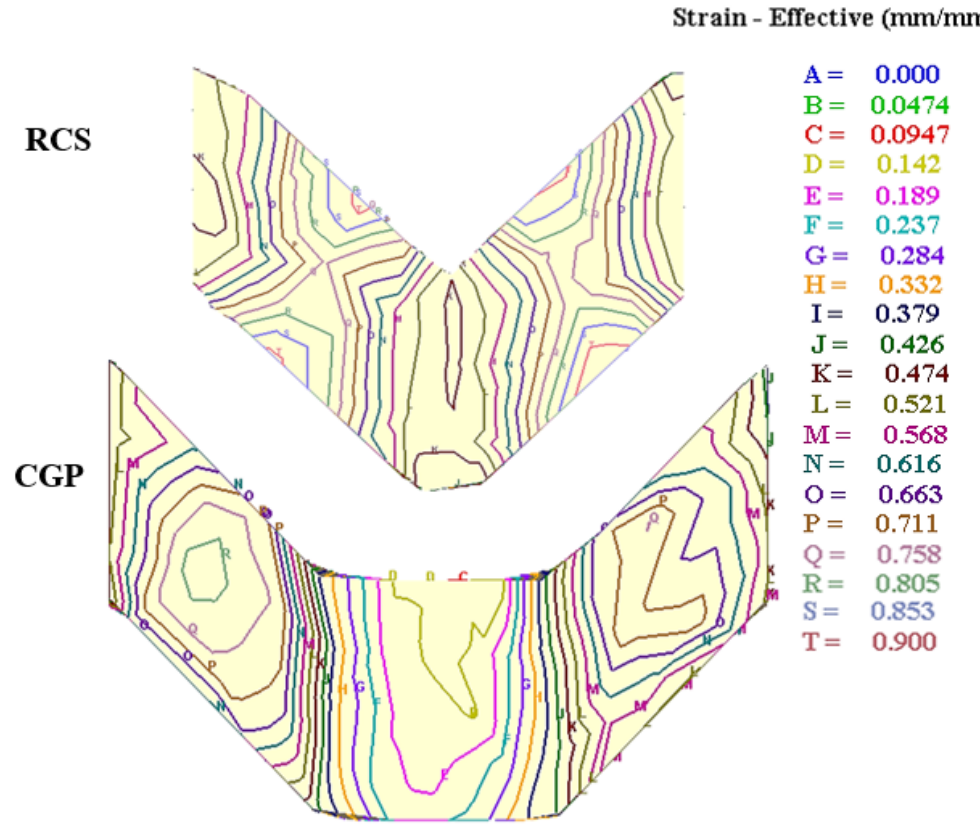

In order to study the distribution of imposed deformation on the processed work-piece after one complete pass of deformation in CGP and RCS, the effective strain was evaluated alongside point A to B for 100 points as shown in Figure 11(a). The effect of CGP and RCS on the effective strain distribution is illustrated in Figure 11(b). As shown in this figure, the sample processed by CGP experiences more effective plastic strain compared to RCS. The reason can be attributed to applied deformation in a pure shear pattern, as in CGP process, the sample separately undergoes shear deformation and flattening in two different cycles. As a result, the work-piece takes higher stress which results in accumulation of higher values of strain. 
Figure 11 (a) Schematic illustration of point A to B for variation of effective strain for hundred points (b) Effective strain distribution of deformed work-piece in CGP and RCS (see online version for colours)

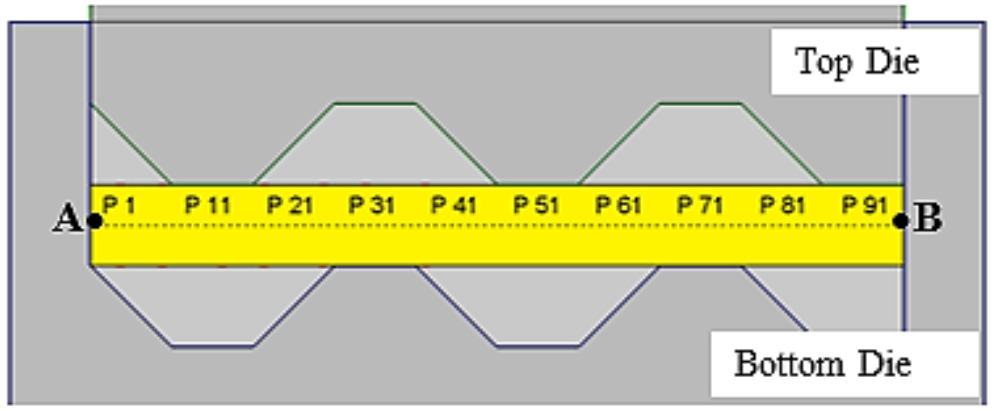

(a)

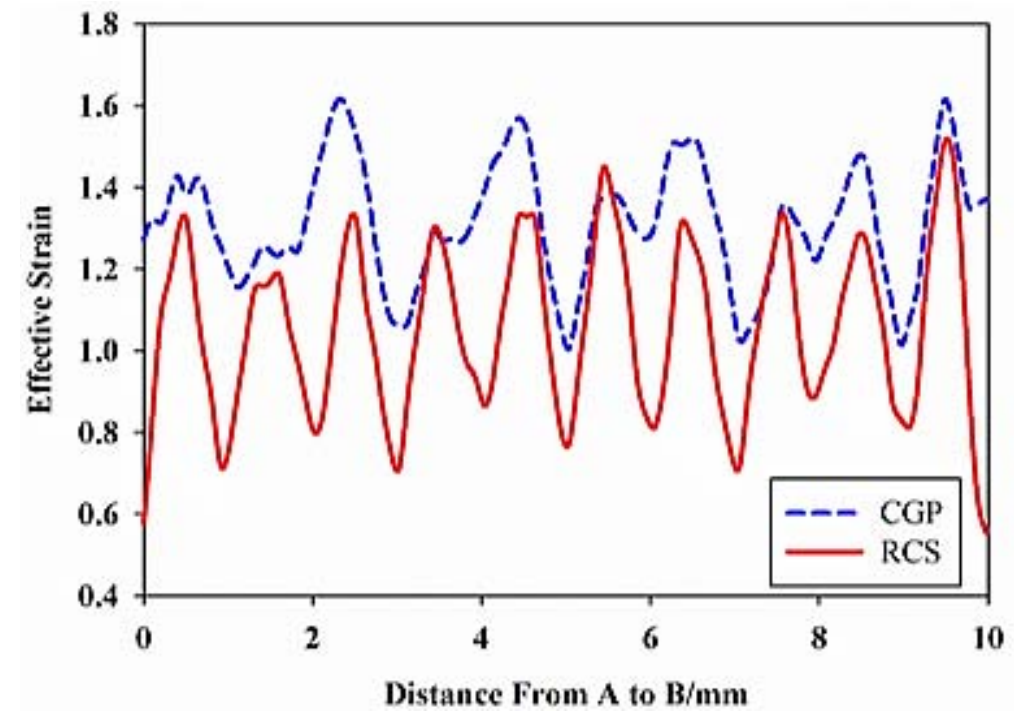

(b)

Figure 12 Inhomogeneity index of deformation in CGP and RCS after one pass of deformation

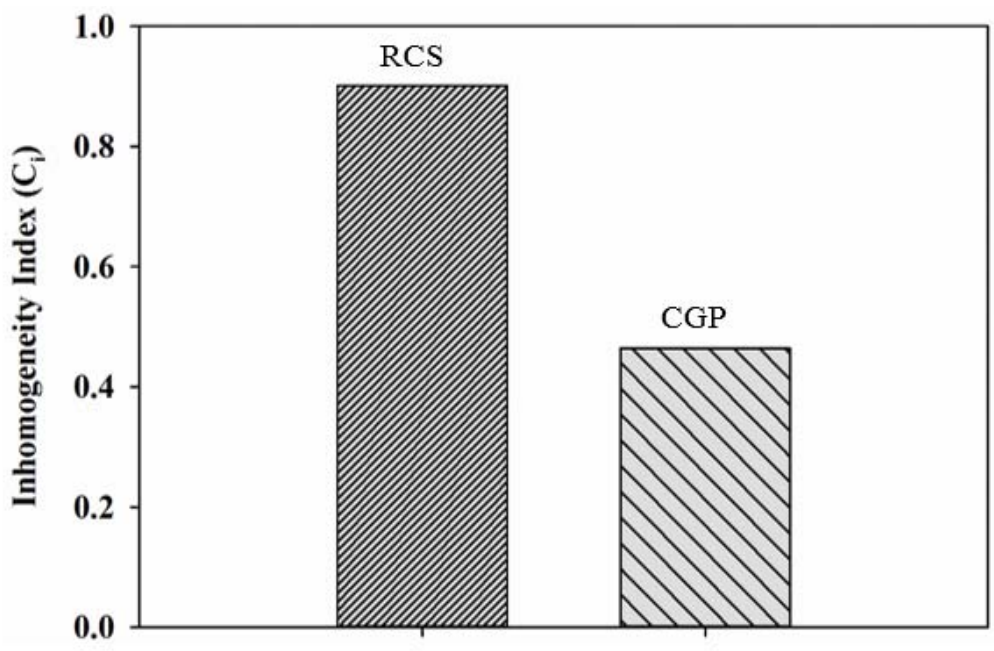

Figure 12 shows the inhomogeneity index of effective strain distribution along line AB. One may see that in this figure, the inhomogeneity of the effective strain imposed to the sample in RCS is higher than CGP. Thus, the work-piece is deformed more 
heterogeneously in RCS and the mechanical properties of the processed sample is distributed uneven which is already explained above. However, the deformed work-piece in CGP shows better distribution of the effective strain with little fluctuation as shown in Figures 11(b) and (12). This is in line with the results that are reported by Sajadi et al. (2012). It should be noted that in all SPD techniques it is a key factor to deform the work-piece homogenously.

\subsection{Process effect on the applied load}

Figure 13 shows the variation of the pressing load versus time during deformation in CGP and RCS. According to the figure, the amount of required load during deformation in RCS is relatively higher than CGP. This is related to the frictional condition between the surfaces of the work-piece and the die walls during deformation. One may see that in RCS a large portion of the sample is imposed to shear deformation, thus, it consume higher frictional energy in the course of deformation. On the other hand, in CGP, a portion of the sample endures no deformation and as a result it does not consume such amount of frictional energy in comparison with RCS. It should be noted that the presence of these peaks in Figure 13, demonstrate the end of each cycles of the processing in CGP and RCS.

Figure 13 The effect of process on the pressing load (see online version for colours)

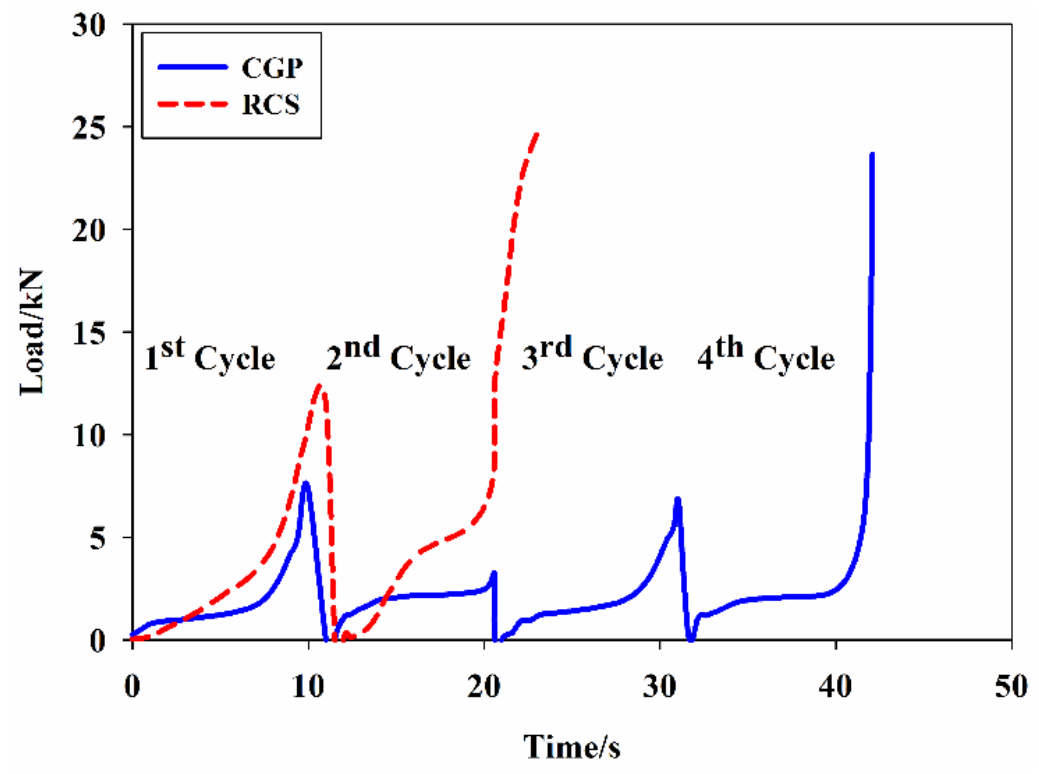

\section{Conclusions}

In the present study, the effects of RCS and CGP on the deformation behaviour of a pure aluminium sheet are investigated using FEA. Based on the FEA results, the following conclusions are made:

1 Filling ratio of the die in CGP is higher than RCS. Consequently, it is possible to repeat the process for more than one cycle to achieve high values of effective strain which results in high level of grain refinement. 
2 The deformed sample processed by CGP endures higher values of effective strain than RCS. Moreover, the effective strain is imposed more uniformly on the sample in CGP so the inhomogeneity index in this process is less than RCS.

3 The FEA results show a good agreement with those in experiment conducted by Ghazani and Vajd (2014) that indicate the accuracy of the computer simulation. In addition, it shows that the implemented boundary conditions in the simulation are as close as with those in experiments.

\section{References}

Bisadi, H., Rastgou, A., Payganeh, G.H., Zeynali, E. and Ghahremani, A. (2010) 10th Iran. Conf. Manuf. Eng., Babol, Iran.

Ghazani, M.S. and Vajd, A. (2014) 'Finite element analysis of the groove pressing of aluminum alloy', Model. Numer. Simul. Mater. Sci., Vol. 4, pp.32-36.

Hosford, W.F. and Caddell, R.M. (1993) Metal Forming-Mechanics and Metallurgy, PTR Prentice Hall, Englewood Cliffs, NJ.

Huang, J.Y., Zhu, Y.T., Jiang, H. and Lowe, T.C. (2001) 'Microstructures and dislocation configurations in nanostructured $\mathrm{Cu}$ processed by repetitive corrugation and straightening', Acta Mater., Vol. 49, pp.1497-1505.

Khodabakhshi, F., Kazeminezhad, M. and Kokabi, A.H. (2010) 'Constrained groove pressing of low carbon steel: nano-structure and mechanical properties', Mater. Sci. Eng. A, Vol. 527, pp.4043-4049.

Li, S., Bourke, M., Beyerlein, I., Alexander, D. and Clausen, B. (2004) 'Finite element analysis of the plastic deformation zone and working load in equal channel angular extrusion', Mater. Sci. Eng. A, Vol. 382, pp.217-236.

Saito, Y., Tsuji, N., Utsunomiya, H., Sakai, T. and Hong, R. (1998) 'Ultra-fine grained bulk aluminum produced by accumulative roll-bonding (ARB) process', Scr. Mater., Vol. 39, pp.1221-1227.

Sajadi, A., Ebrahimi, M. and Djavanroodi, F. (2012) 'Experimental and numerical investigation of Al properties fabricated by CGP process', Mater. Sci. Eng. A, Vol. 552, pp.97-103.

Scientific Forming Technologies Corporation (2006) User's Manual, DEFORM, TM, 3D Version 6.1 (sp1), Columbus $\mathrm{OH}$.

Talab, A.R.R. and Ghalandari, L. (2011) Iran. Conf. Recent Tech. Mech. Eng., Shiraz, Iran.

Valiev, R.Z., Islamgaliev, R.K. and Alexandrov, I.V. (2000) 'Bulk nanostructured materials from severe plastic deformation', Prog. Mater. Sci., Vol. 45, pp.103-189.

Zhu, Y.T., Lowe, T.C., Jiang, H. and Huang, J. (2001) Method for Producing Ultrafine-grained Materials using Repetitive Corrugation and Straightening, US Patent No. 6197129, US Patent and Trademark Office, Washington, DC. 